\title{
A SIX-LEGGED MEMS SILICON ROBOT USING MULTICHIP ASSEMBLY
}

\author{
Daniel S. Contreras, and Kristofer S. J. Pister
}

Berkeley Sensor and Actuator Center, Berkeley, California, USA

\begin{abstract}
A silicon-based six-legged robot was designed, fabricated, and tested. The robot is based on legs using electrostatic inchworm actuators with force densities of $1.8 \mathrm{mN} / \mathrm{mm}^{2}$ at $100 \mathrm{~V}$, a $62 \%$ improvement over similar designs previously demonstrated when normalized to $\mathrm{V}^{2}$. These motors manipulate planar silicon linkages, fabricated alongside the motors in a two-mask silicon-on-insulator (SOI) process. Three of these legs are arranged to actuate off the edge of their fabricated chip. Using pillars with matching throughholes, two of these leg chips are assembled with a central base for mechanical support and electrical routing, totaling 12 motors, 6 linkages, and 12 degrees-of-freedom on the robot. The 3D MEMS assembly process successfully routes 24 separate signals to wires for external control. The robot weighs $200 \mathrm{mg}$ and measures $9.6 \mathrm{~mm}$ wide, $7 \mathrm{~mm}$ tall, and $13 \mathrm{~mm}$ long. The robot has walked forward under tethered operation.
\end{abstract}

\section{INTRODUCTION}

Many actuation schemes have been developed for millimeter to centimeter scale terrestrial robots, such as external electric and magnetic fields [1], [2], thermal actuators [3], piezoelectric actuators [4], [5], and electrostatic actuators [6], [7]. Comparing these platforms, robots controlled by external field are dependent on a field source and have limited autonomy. Thermal actuator robots are costly in terms of power draw and have low actuator bandwidth leading to slower operation. Piezoelectric actuator based robots are comparable to electrostatic actuators in terms of power draw and force output, but can have complicated fabrication processes and limited range of travel.

Electrostatic actuators were chosen for this robot due to their low power draw, high force output, long travel and simple fabrication. A single-legged silicon-based robot was previously demonstrated in [8]. This robot relied on electrostatic gap closing actuator (GCA) array based angled-arm inchworm motors based on work in [9]. These motors demonstrated force densities of $0.5 \mathrm{mN} / \mathrm{mm}^{2}$ and have been shown to reach unloaded shuttle speeds of over $30 \mathrm{~mm} / \mathrm{s}$ [10]. The motors manipulated a silicon linkage that actuated off the edge of the chip and had a swept area of approximately $0.5 \mathrm{~mm} \times 0.5 \mathrm{~mm}$. The robot could walk while connected using long wires to a control and power source.

The robot presented in this work takes a similar version of this leg and uses it as a unit cell for a six-legged robot. Figure 1 shows the robot standing upright. This robot is composed of three separate chips; two leg chips and a top hub chip. Using hand assembly, the robot is constructed from these discrete pieces. It is externally wired to a control and power source through pads on the hub chip, which route the actuator signals from the leg chips.

\section{ROBOT DESIGN}

\section{Planar Design Methodology}

The design for these robots focuses on using entirely planar mechanisms to develop mobility mechanisms. This takes advantage of the fact that MEMS technology is ideal for fabricating planar mechanisms. It also has the advantage of lending itself to easier fabrication design than previous robots that use complex multi-mask processes to develop out-of-plane joints or depositing piezoelectric materials on complicated topographies. The obvious limitation is

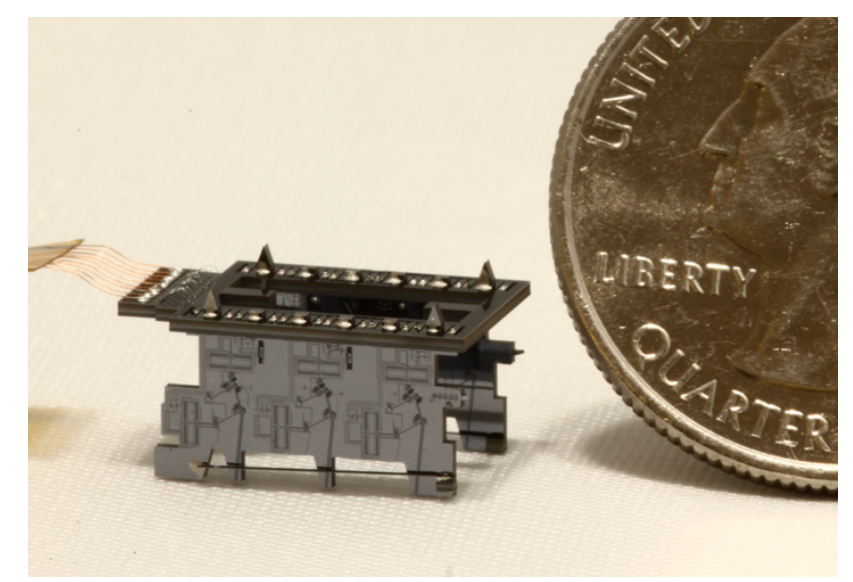

Figure 1: An assembled robot standing upright next to a US quarter

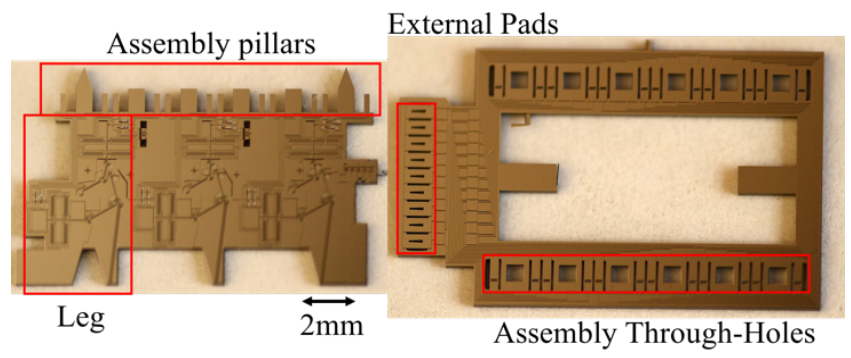

Figure 2: The leg chip, highlighting the leg unit cell and the pillars (left) The hub chip highlighting the assembly throughholes and the pads for external wiring (right)

that the mechanisms designed in this process can have at most two degrees of freedom.

In order to overcome the planar limitation and actuate on the ground, the robots are designed to extend their legs off the edge of the chip substrate. The entire chip is then flipped on its side to allow for ground actuation. In this way, complex processing is forgone in favor of post-fabrication assembly.

\section{Leg Chip}

The leg chip is shown in Figure 2. The leg chips have two main components, the legs and the assembly/routing pillars.

The leg linkage design is similar to the design shown in [8]. The stride length was modified to allow for a longer horizontal sweep when the leg is on the ground. The current stride length is $1 \mathrm{~mm}$, twice what was demonstrated before. The linkage also uses newly designed rotary springs. These springs were designed to maintain a constant rotational stiffness through the angular travel of the joint.

Two motors are attached to the leg linkage; one for vertical axis actuation and the other for horizontal axis actuation. The placement of the vertical axis motor was designed to be in-line with the end of the leg linkage for a 1:1 mechanical advantage. This is done in order to maximize the force output as the robot lifts itself. The horizontal axis motor was placed with a 4:1 mechanical advantage, dropping the motor's force output in favor of speed during the robot's horizontal stride. Each leg has 2 degrees-of-freedom, granting the 


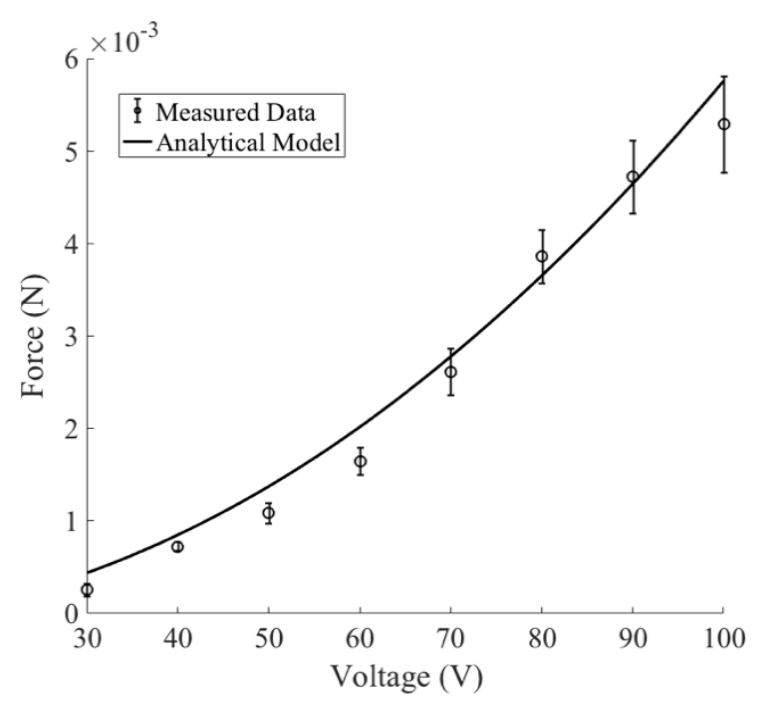

Figure 3: The raw force output of an electrostatic inchworm motor used on the leg chip. At $100 \mathrm{~V}$ the motor has a force output of approximately $5 \mathrm{mN}$

robot 12 degrees-of-freedom when fully assembled.

The raw force output of the motor is shown in Figure 3. In order to increase the force density of the motor to lift the mass of the robot, the motors were designed using a drawn $1 \mu \mathrm{m}$ feature size between the pawls and the shuttle of the motor. The force was measured using a spring gauge attached to the shuttle. The spring gauge was calibrated using an array of comb drive resonators. The total layout area of the motor measures $2.7 \mathrm{~mm}^{2}$. This leads to a higher force density of approximately $1.85 \mathrm{mN} / \mathrm{mm}^{2}$ while actuating at $100 \mathrm{~V}$. Normalizing to $\mathrm{V}^{2}$, this is a $62 \%$ improvement over the previous maximum force density of $1.38 \mathrm{mN} / \mathrm{mm}^{2}$ at $110 \mathrm{~V}$ [9].

The assembly pillars are shown at the top of the leg chip. The motor signals are routed through wirebonds and silicon to terminate at the pillars. Each motor requires two signals and ground, meaning a single leg requires four signals and a ground. Each leg chip routes 12 signals and ground to the pillars. The ground for each motor is routed to a separate pillar, leaving 18 pillars in total.

The pillars for the high voltage motor signals have the substrate etched from behind them, leaving them as device layer only. The substrate is left behind the ground pillars, since the ground signal is also routed to the substrate. This way they can also be used as durable assembly guides, to protect the fragile device layer pillars.

\section{Hub Chip}

The hub chip is also show in Figure 2. The hub chip is meant to fit on top of two adjacent leg chips using a pillar/through-hole assembly technique adapted from work shown in [11].

The purpose of the hub chip is two-fold. First, the hub chip is meant to hold the leg chips upright, such that they can actuate on the ground. Second, the hub chip routes electrical signals from the legs on the leg chip to pads that interface externally with long wire tethers. The robot routes the signals from the legs to demonstrate a dual tripod gait on the robot.

The designed gait and walk cycle of the legs is shown in Figure 4. This gait is hardwired into the routing of the hub chip. For the robot, three legs are actuated in tandem while the remaining set of three legs are actuated out of phase. This gait was chosen because it is statically stable. At any given time, three points around the robot's center of mass are always in contact with the ground. (a)

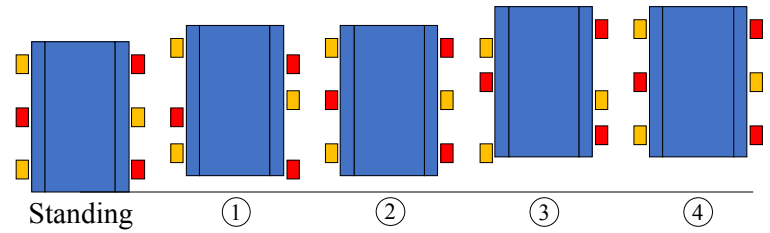

(b)

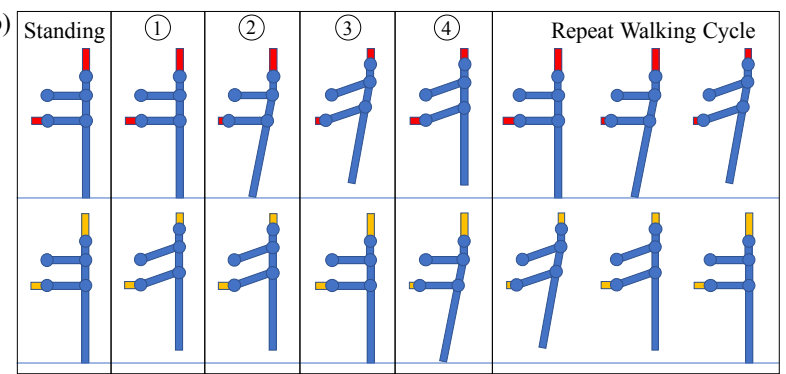

Figure 4: (a) The tripod gait of the robot is shown here. The red squares represent one set of three legs that are actuated in tandem while the orange squares represent the other tripod actuated out of phase with respect to the first set (b) A diagram showing the actuation of the legs. The position of the motors is represented by the colored blocks. The color of the motors and the numbering above the legs matches the colors and numbering in the 5 a to correspond to the state of the leg during the robot's state of motion

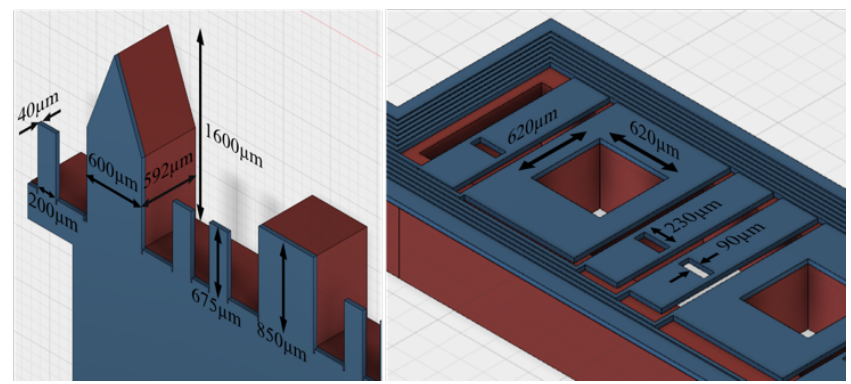

Figure 5: CAD model of chips, detailing dimensions of the pillars (left) and the holes on the hub chip (right)

Pillar Design and Tolerance

Figure 5 details the design and dimensions of the pillars and the holes. There are three types of pillars used in this design; routing pillars for routing the high voltage signals made up of only device layer silicon, square block pillars made of device layer silicon and substrate silicon for mechanical rigidity and to protect the routing pillars, and triangular alignment pillars to align the holes and to gently guide the hub chip into place on the leg chips.

The alignment and block pillars share the same hole size. The hole on the hub chip is designed to have $10 \mu \mathrm{m}$ of tolerance on a given side around the pillars, factoring in the uncertainty in the thickness of the substrate. For the routing pillars, the tolerance is set to $15 \mu \mathrm{m}$ on a given side and $25 \mu \mathrm{m}$ in the dimension of the wafer thickness to account for uncertainty in the substrate thickness. With this larger tolerance, the alignment and block pillars protect the routing pillars as the hub chip falls into place.

\section{FABRICATION AND ASSEMBLY}

\section{Processing}

All the parts of the robot are fabricated in a two-mask siliconon-insulator (SOI) process. The SOI wafers have a $40 \mu \mathrm{m}$ device 

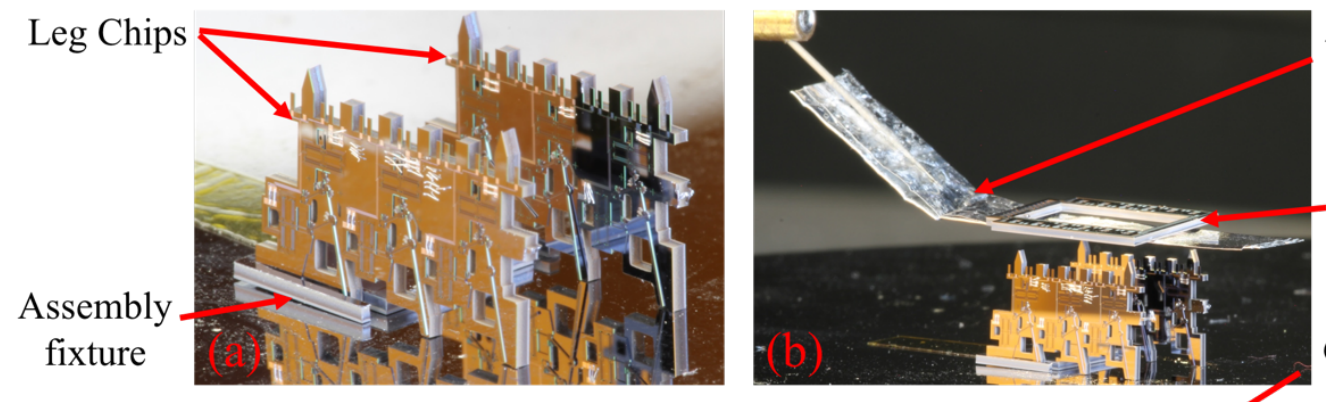

Aluminum
Foil Lift
Hub Chip
Guide pillar,
ground
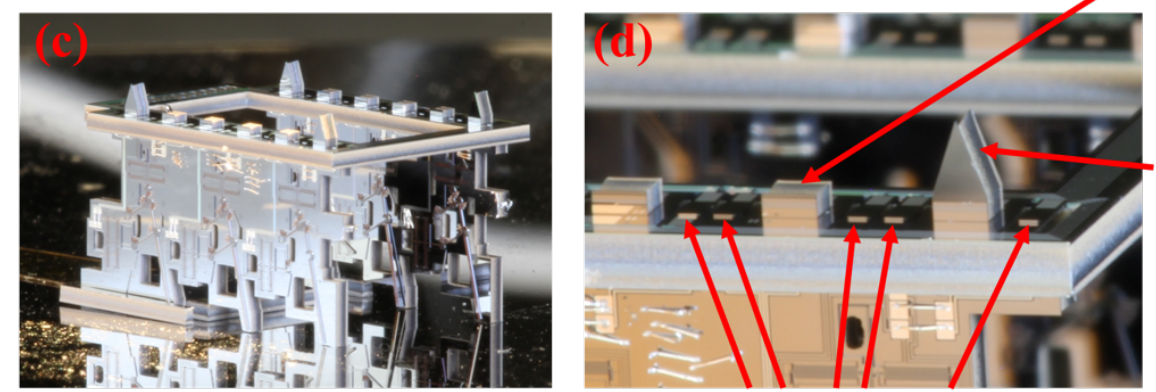

Device layer pillars,
motor signals

Figure 6: The assembly of the robot (a) The leg chips are held upright in the assembly fixture and are placed underneath a microscope at a probe station (b) The hub chip is placed onto an aluminum foil lift that is glued to a probe tip on a micromanipulator. The holes in the hub chip are aligned to the pillars on the leg chips through the microscope (c) The hub chip is dropped into place and the aluminum lift is removed (d) The pillars are visible through the holes of the hub chip. After the structures are placed correctly, silver epoxy is used to join the pillars and the base around the holes on the hub chip. The assembly is then cured on a hotplate

layer, $2 \mu \mathrm{m}$ buried oxide, and a $550 \mu \mathrm{m}$ substrate. The robot legs, hub chip, and assembly base are patterned into the layer of device layer silicon. The wafer is temporarily bonded to a handle wafer and an etch is performed through the backside of the wafer to reduce the mass of the robot and singulate each of the individual pieces. The pieces are etched in a vapor-phase anhydrous HF etch to release moving parts from the substrate. Once the pieces are released, the chips are wirebonded to route motor signals to the pillars on the leg chips and to route signals to the pads for external connection on the hub chip. The wirebonds are visible in Figure 7 (b) and (c).

\section{Assembly}

The assembly process is shown in Figure 6. In addition to the robot chips, additional structures for the purpose of assembly are also fabricated. This includes an assembly base, used to hold the leg chips upright during assembly and a bracket piece to place over the legs to keep ensure they do not come out of plane during actuation. The base is shown in Figure 6 and the leg bracket is shown in Figure 9. The leg chips are manually placed into the assembly base such that they stand upright. This structure is placed at a probe station where a microscope can be used for alignment.

A lift made from folded aluminum foil is glued onto a probe tip which is inserted into a micromanipulator for precision in alignment. The hub chip is manually placed on the lift and positioned above the leg chips. Alignment is performed through the microscope. The holes in the hub chip are positioned such that they line up with the pillars on the leg chips. Once the positioning is correct the hub chip is dropped with the micromanipulator to sit on the leg chips.

The assembly is permanently affixed using silver epoxy to join the pillars to the base of the holes. The epoxy also electrically routes the motor signals between legs that make up each of the tripods and to the hub chip pads. The silver epoxy joints on the pillars are shown in Figure 8.
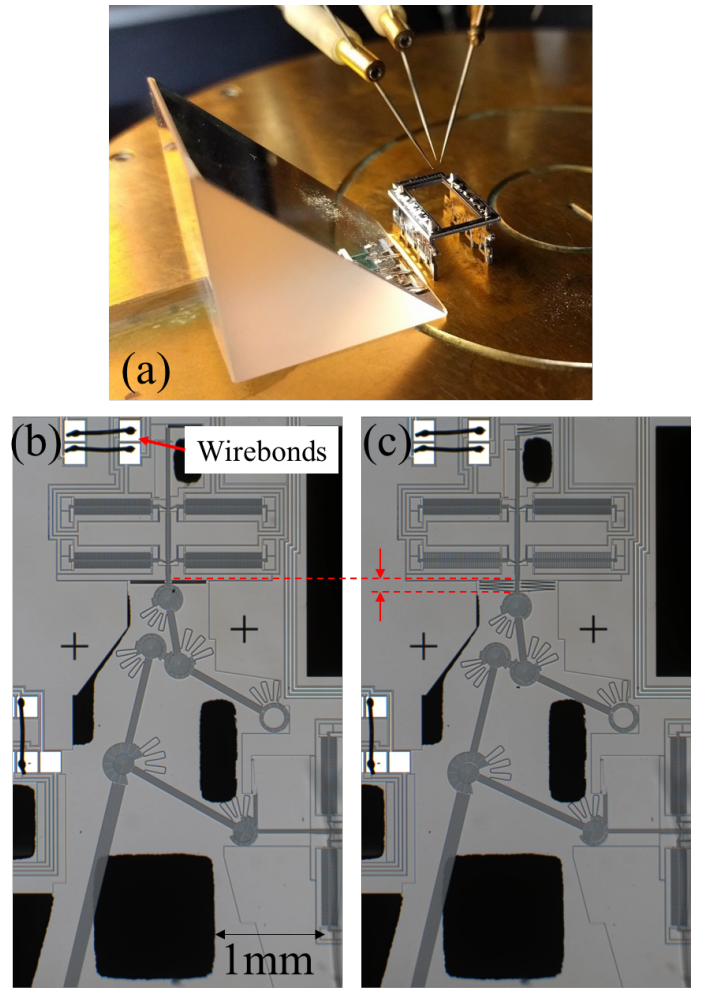

Figure 7: (a) Signals are delivered to the hub chip via probes (b) The unactuated leg seen through the microscope objective off of a 45-degree mirror (c) The leg actuating. The red lines indicate the displacement 


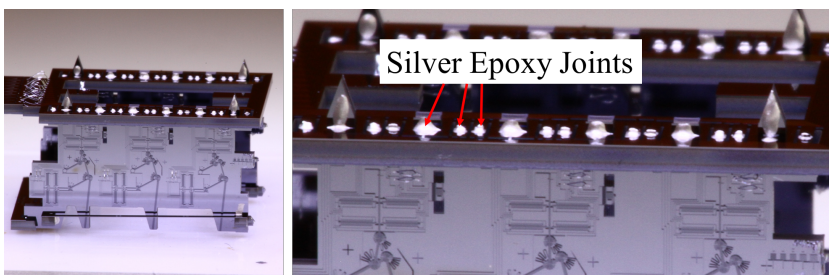

Figure 8: Silver epoxy joints connecting the hub chip to the leg chips

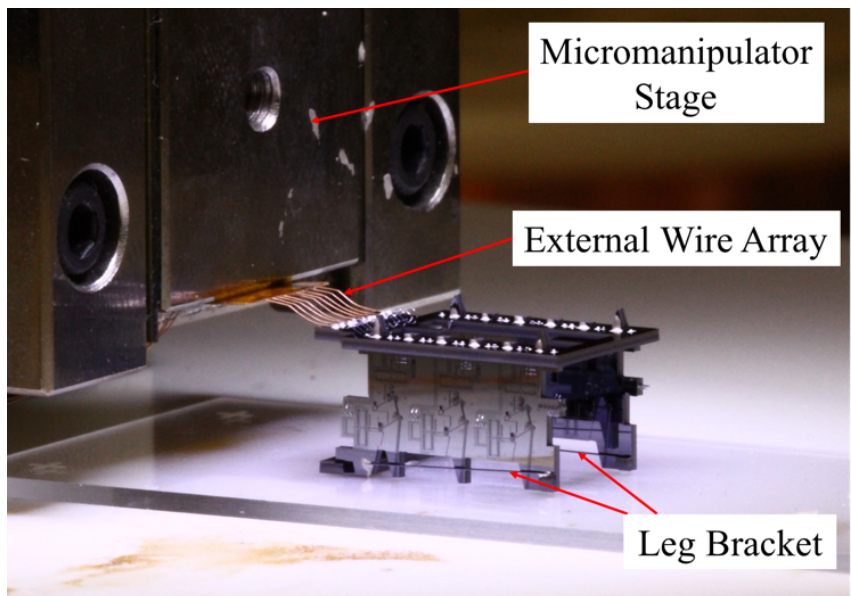

Figure 9: Wiring the robot using a micromanipulator stage

\section{Signal Verification}

Once the robot is assembled and the epoxy is cured, it is placed under the probe station to test the connections. A leg actuating through signals to the hub chip is shown in Figure 7. Probes are placed on the hub chip pads to drive each of the motors. A 45-degree mirror is used to inspect the actuation. The silver epoxy on the pillars successfully routes the signals to the correct GCA's through visual inspection of the pull-in voltage.

\section{Wiring}

The hub chip has an array of pads extending off the rear of the chip. External wires are connected to these pads. Figure 9 shows the process of wiring-up a robot. An array of $30 \mathrm{~cm}$ long $60 \mu \mathrm{m}$ thick copper wires are aligned in an array to line up with the pitch of the pads. The ends of the wires are dipped in silver epoxy. The array is held by a micromanipulator and positioned above the pads through a microscope. The wires are than dropped into position on the pads. Once the tips of the wires make contact with the pads, the assembly is cured on a hotplate to cure the epoxy and affix the wires. The other ends of the wires are soldered to a header pin which is inserted into the robot control circuit. After curing, the now wiredup robot can be detached from the micromanipulator.

\section{WALKING}

Once the robot is assembled and wired it is placed on a flat surface to walk. Figure 10 shows an assembled robot taking steps. The robot takes two full steps in the sequence shown. This robot had three functioning legs and was able to walk forward using two of the legs which were joined in the dual-tripod actuation scheme. The actuation voltage was $100 \mathrm{~V}$

The velocity of the horizontal stride of each step was measured. The first step was $7.5 \mathrm{~mm} / \mathrm{s}$ and the second step was $1 \mathrm{~mm} / \mathrm{s}$. The motors were actuated at $100 \mathrm{~Hz}$, which translates to a horizontal speed of approximately $1 \mathrm{~mm} / \mathrm{s}$. The first step was faster than

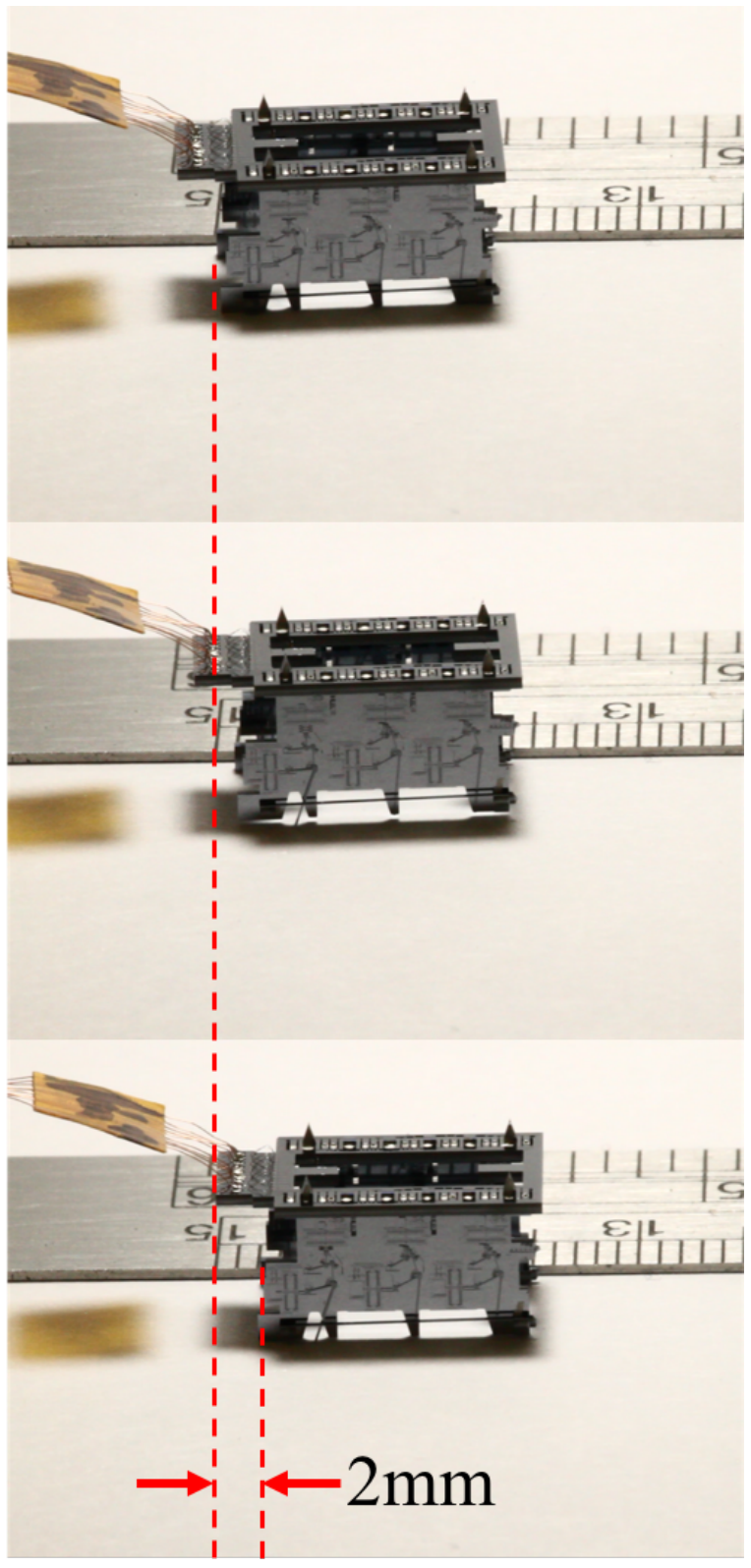

Figure 10: The robot taking steps. In this sequence, the robot takes two steps. Each step moves the robot forward by $1 \mathrm{~mm}$. The robot was ultimately limited by tension in the wires and running against the ruler

expected. This could be attributed to the weight of the body carrying the robot forward under the influence of gravity. The robot is designed to drop its mass as it takes its step.

The total capacitive load of a single actuator is estimated to be $26 \mathrm{pF}$ when the gaps are fully closed. Operating at $100 \mathrm{~Hz}$ at $100 \mathrm{~V}$ this leads to a power draw of $78 \mu \mathrm{W}$ for a single tripod.

In addition to walking forward as designed, the robot also demonstrated repeatable unintentional locomotion schemes. When the leg bracket was not put in place the legs of the robot would splay out of plane upon contact with the ground, and would move the robot to the side, like a crab. The legs would return to their initial position without breaking.

With top motor speeds of $34 \mathrm{~mm} / \mathrm{s}$ demonstrated in [10] and a 4:1 mechanical advantage, the robot could potentially demonstrate 


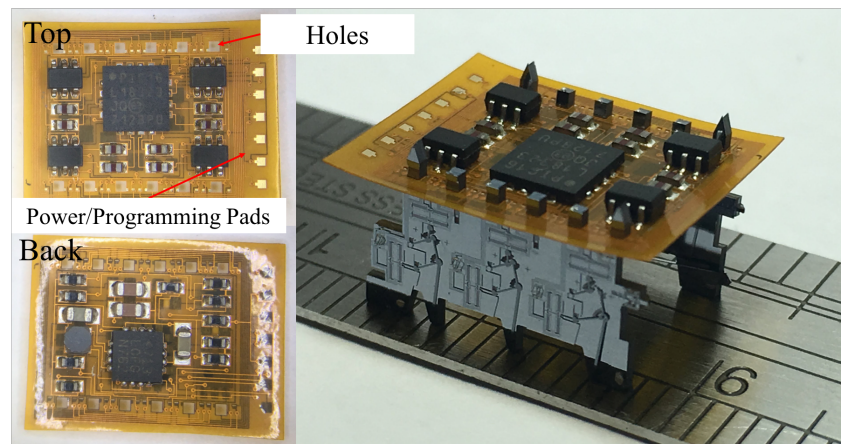

Figure 11: The flexible circuit board. The board has holes that are meant to interface with the pillars on the leg chips in the same way as the hub chip. The top and back of the board are shown. The top has the microcontroller and high voltage transistors while the back of the board has the boost-converter circuit. On the right, a populated board is shown mounted onto the larger pillars of a set of leg chips

top speeds of over $13.6 \mathrm{~cm} / \mathrm{s}$.

\section{FLEXIBLE CIRCUIT BOARD BACKPACK}

In addition to the robot design made entirely of silicon, we have also done work on creating a robot controller and motor driver on a lightweight flexible circuit board substrate, hereby referred to as the robot backpack. The purpose of the robot backpack is to use the most lightweight commercial-off-the-shelf (COTS) and create an on-board controller that is within the robot's payload.

The circuit board is shown in Figure 11. The circuit's mass is approximately $170 \mathrm{mg}$. The backpack operates entirely on a $3.7 \mathrm{~V}$ source. It uses an LT3482 boost DC/DC converter to generate a $90 \mathrm{~V}$ signal from the $3.7 \mathrm{~V}$ source. The high voltage signal is used as the high voltage rail for a network of high voltage transistors through pull-up resistors. These transistors are switched by a PIC16(L)F183XX microcontroller, which was chosen due to mass considerations. The board is meant to generate the high voltage signals for a dual tripod gait. With this board, the number of external connections drops from nine to two after the microcontroller has been programmed. The board has successfully generated an $88 \mathrm{~V}$ signal that can drive the inchworm motors.

The robot flex backpack has the same array of holes that are on the hub chip. The backpack is meant to interface to the leg chips in the exact same way that the hub chip interfaces to the leg chips. The flexible circuit board was patterned with a $2 \mu \mathrm{m}$ feature size in order to reduce the total size of the board.

\section{CONCLUSION}

We have demonstrated successful actuation and steps taken of a six-legged silicon robot made of multiple chips, actuated through long tethers. The robot is able to move forward using only three of its six legs. The multichip assembly process provides reliable routing for the 3D MEMS structure, from the top hub chip to the side leg chips.

Current issues to address with the robot focus on yield and reliability of operation after assembly. Stiction frequently occurs during assembly and while the devices are in operation. This has been attributed to how sensitive the legs are to electrostatic forces and charging causing the legs to snap down and stick to the substrate. Preliminary experiments with self-assembled monolayers, such as FOTS, have shown promising results for preventing in operation stiction. Reduction of the substrate area can also be beneficial for stiction as well as reducing the robot's total mass.

\section{REFERENCES}

[1] D. Vogtmann, R. S. Pierre, and S. Bergbreiter, "A $25 \mathrm{mg}$ magnetically actuated microrobot walking at $>5$ body lengths/sec," in Micro Electro Mechanical Systems (MEMS), 2017 IEEE 30th International Conference on, Las Vegas, Nevada, USA, 2017, pp. 179-182.

[2] B. R. Donald, C. G. Levey, C. D. McGray, I. Paprotny, and D. Rus, "An untethered, electrostatic, globally controllable MEMS micro-robot," J. Microelectromechanical Syst., vol. 15, no. 1, pp. 1-15, 2006.

[3] K. Saito, K. Iwata, Y. Ishihara, K. Sugita, M. Takato, and F. Uchikoba, "Miniaturized Rotary Actuators Using Shape Memory Alloy for Insect-Type MEMS Microrobot," Micromachines, vol. 7, no. 4, p. 58, Mar. 2016.

[4] A. T. Baisch, O. Ozcan, B. Goldberg, D. Ithier, and R. J. Wood, "High speed locomotion for a quadrupedal microrobot," Int. J. Robot. Res., vol. 33, no. 8, pp. 10631082, 2014.

[5] J. Choi et al., "Thin-film piezoelectric and high-aspect ratio polymer leg mechanisms for millimeter-scale robotics," Int. J. Intell. Robot. Appl., vol. 1, no. 2, pp. 180-194, Jun. 2017.

[6] S. Hollar, A. Flynn, C. Bellew, and K. S. J. Pister, "Solar powered $10 \mathrm{mg}$ silicon robot," in Micro Electro Mechanical Systems (MEMS), 2003 IEEE 16th International Conference on, Kyoto, Japan, 2003, pp. 706-711.

[7] M. Qi, Y. Zhu, Z. Liu, X. Zhang, X. Yan, and L. Lin, “A fastmoving electrostatic crawling insect," in Micro Electro Mechanical Systems (MEMS), 2017 IEEE 30th International Conference on, Las Vegas, Nevada, USA, 2017, pp. 761-764.

[8] D. S. Contreras, D. S. Drew, and K. S. Pister, "First steps of a millimeter-scale walking silicon robot," in Solid-State Sensors, Actuators and Microsystems (TRANSDUCERS), 2017 19th International Conference on, 2017, pp. 910-913.

[9] I. Penskiy and S. Bergbreiter, "Optimized electrostatic inchworm motors using a flexible driving arm," $J$. Micromechanics Microengineering, vol. 23, no. 1, p. 015018, Jan. 2013.

[10] D. S. Contreras and K. S. Pister, "Dynamics of electrostatic inchworm motors for silicon microrobots," in Manipulation, Automation and Robotics at Small Scales (MARSS), 2017 International Conference on, 2017, pp. 1-6.

[11] G. Xue, M. Toda, and T. Ono, "Comb-Drive XYZ-microstage With Large Displacements Based on Chip-Level Microassembly," J. Microelectromechanical Syst., vol. 25, no. 6, pp. 989-998, Dec. 2016.

\section{CONTACT}

*D.S. Contreras, dscontreras@berkeley.edu 